\title{
Immediate Implant Placement in Anterior Extraction Sockets using a Synthetic Putty as Graft: A case series
}

\author{
Maarten Boogaard ${ }^{1}$ \\ ${ }^{1}$ Affiliation not available
}

November 22, 2021

\author{
Maarten J. Boogaard DMD ${ }^{1}$ \\ ${ }^{1}$ Private Practice: Churchill-laan 162hs, 1078 ER, Amsterdam, The Netherlands \\ - Correspondence: maarten@tandartsboogaard.nl
}

\begin{abstract}
Aim of this case series is to show that synthetic putty as a graft material gives great results in direct implant placement in extraction sockets in the anterior maxilla. Synthetic putty in 1-phase, 2-phase and direct loading cases is a valid alternative for grafting with xeno- or allograft materials.
\end{abstract}

Keywords: Immediate implant placement, bone grafting, synthetic putty, immediate loading, extraction socket.

\section{Introduction}

In the 1980's implant placement in extraction post extraction sockets has been introduced[1]. For more the two decades, the clinical protocol for immediate anterior implant placement into fresh extraction sockets has evolved from a two-stage protocol to a one stage protocol - often flapless. Sometimes with an immediate provisional restoration placed at the same appointment[2].

Most changes in ridge contours happen during the first 12 months post-extraction were predominantly horizontal and more pronounced buccally then lingually[3]. The buccal bone plate is more suspectable to resorption then the lingual plate, due to its thickness[4]. CT scan in 250 patients have showed anterior facial plate thickness ranges from $0.3-1 \mathrm{~mm}$ with about $50 \%$ of the wall thickness is less than $0.5 \mathrm{~mm}$. This suggests that for many patients' extraction of an anterior maxillary tooth will result in loss of the entire buccal plate, changing the ridge contour a lot[5]. The location of the implant, the thickness of the buccal bone crest, and the size of the horizontal buccal gap can significantly influence changes in bone crest after tooth extraction[6].

The mere implant placement into a fresh extraction socket cannot prevent crestal remodeling. However, filling the void between the extraction socket wall and the implant with mineralized collagen bone substitute provides additional amount of hard tissue healing at the entrance of the socket and improved the level of marginal bone- to-implant contact[7]. Immediate loading with a provisional prosthesis is possible, provided that it does not disturb implant osseointegration if the loading forces are well-oriented, and the implant has satisfactory primary stabilization[8].

In these cases, a dental putty can also be used to fill the buccal gap. The dental putty used in these cases is composed of a calcium phosphate silicate trapped in a carrier, it is a bioactive regenerative material that not only acts as an osteoconductive scaffold, but also interact with the surrounding tissues and imparts an osteostimulatory effect ${ }^{(1)}$ The material is ready to be used, and has a transient hemostatic effect designed to provide a comfortable environment for the clinician to work with. It has a great retention and can adapt to the 
defect shape[9]. Most studies indicate that 80-90 percent is absorbed within 4-6 months, while regenerating bone at the same time. The putty has consistently proven to regenerate bone when used for socket grafting, grafting of periodontal defects or in crestal sinus lift procedures[10,11,12,13,14].

\section{Case presentation}

A series of 8 patients presented themselves with single teeth that needed replacement with implants in the maxilla.

\section{Materials and Methods}

8 patients were selected for this case series between the age of 42 to 79 years old. All Patients received $2 \mathrm{~g}$ Amoxicillin and $600 \mathrm{mg}$ Ibuprofen 1 hour before surgery. Chlorhexidine mouth rinse was used 60 seconds before surgery. Surgery was performed using local anesthetics (Ultracain-DS forte).

The protocol of Goene and van Daelen[15] was followed, a flapless atraumatic extraction technique is essential for any anterior maxillary tooth for which immediate implant placement is being considered. After the tooth has been removed, the integrity of the labial plate should be verified with a periodontal probe.

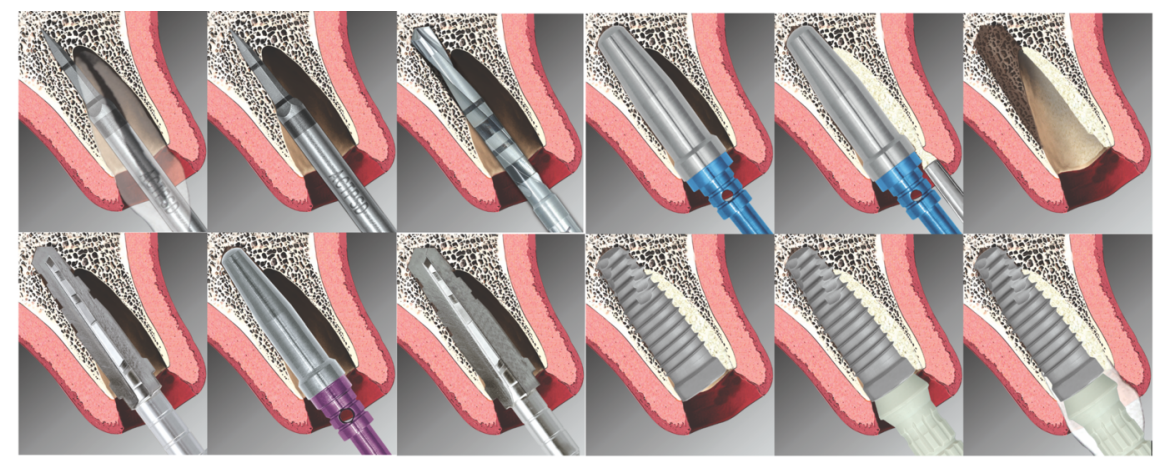

Fig. 1, Schematic overview of protocol

After preparation Biomet 3i ${ }^{(2)}$ implants were placed. All cases were grafted with NovaBone ${ }^{(1)}$ putty $0.25 \mathrm{ml}$ using a locator to separate the putty from the preparation. A Peek healing abutment ${ }^{(2)}$ was used and the temporary crown was made with Protemp $3^{(3)}$ 


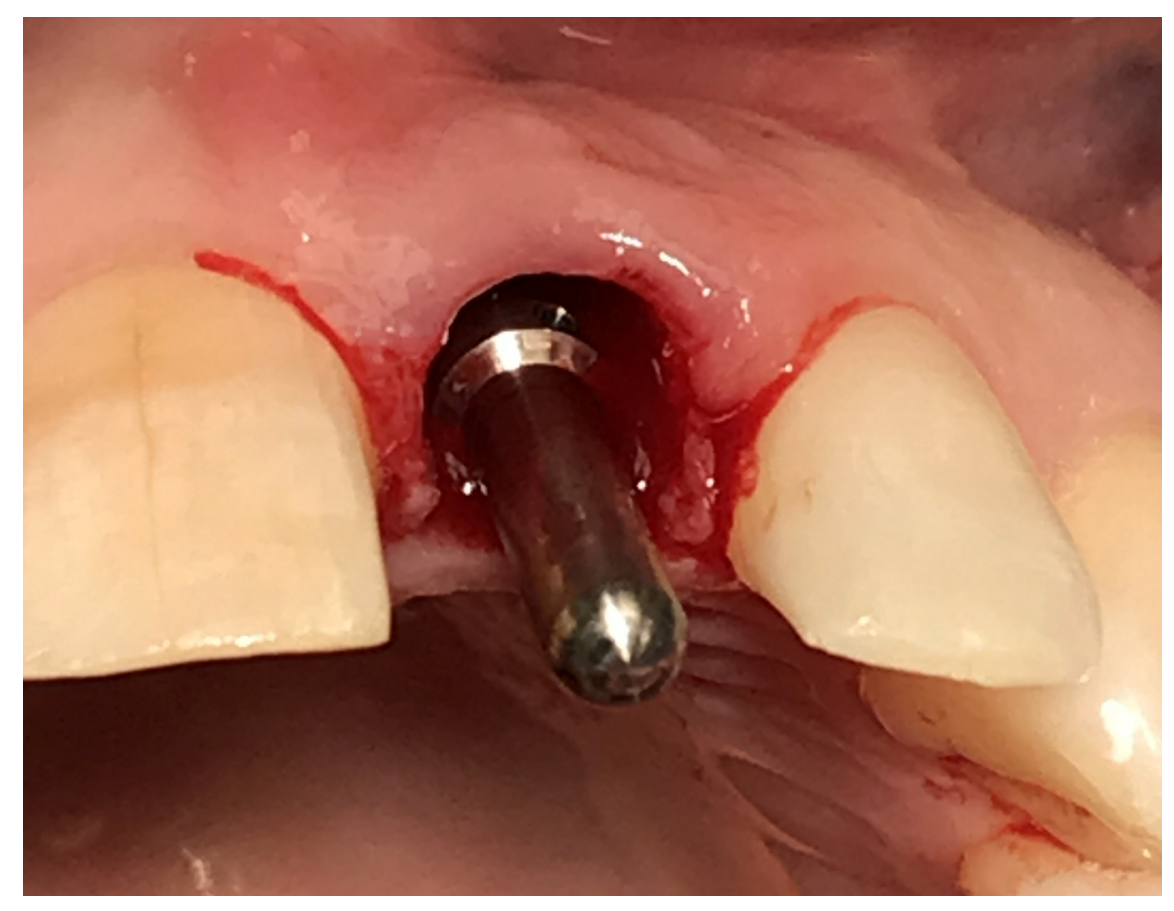




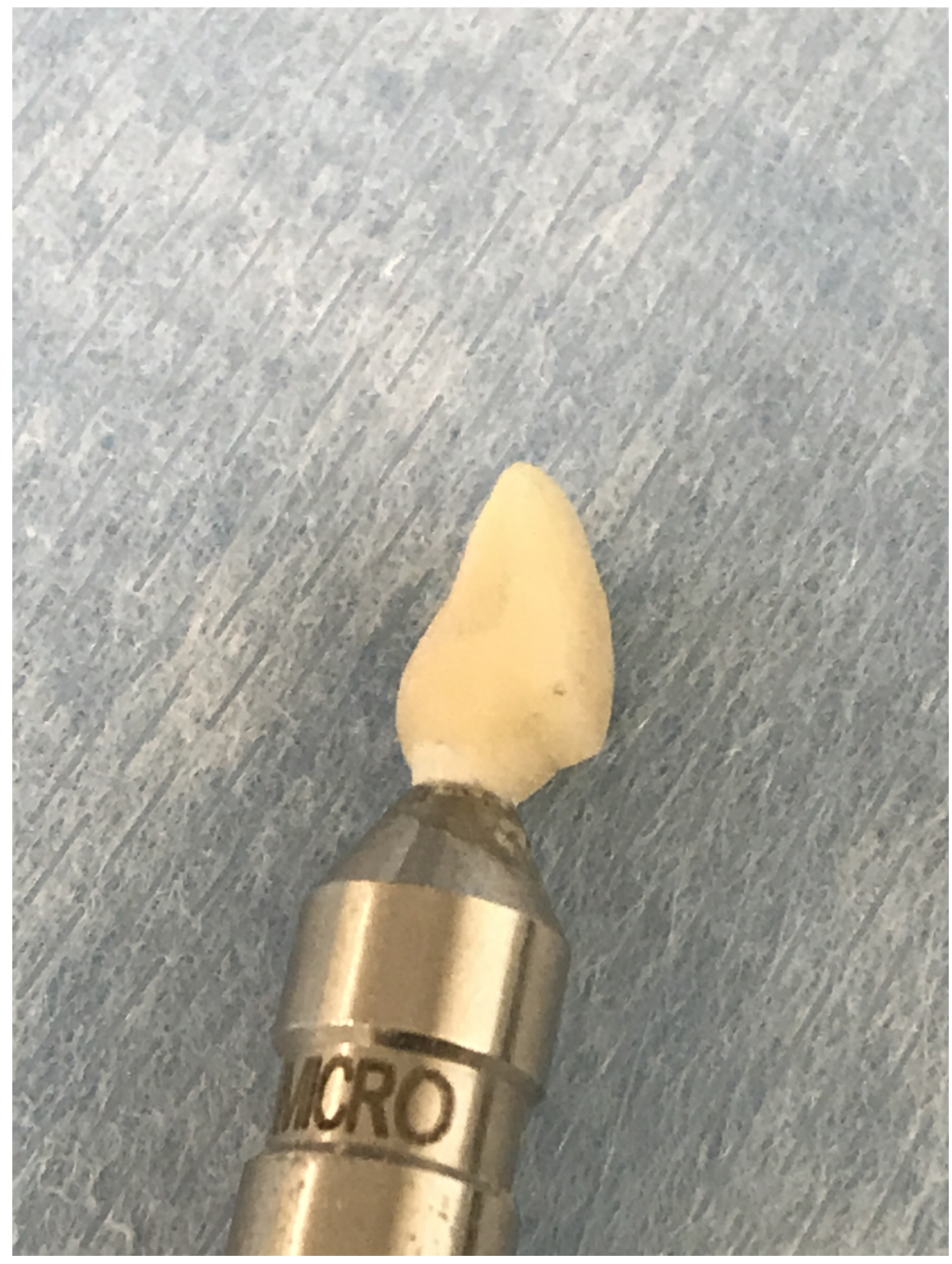

Fig. 2, 3. Placement of direction indicator and application of synthetic putty and temporary crown.

The 1-phase patients received a healing abutment since esthetics was not important for them, and a Bioplug (4) was used to cover the buccal gap. The 2-phase patients received a Osseoguard flex ${ }^{(2)}$ completely covering the implant and extraction site. An example of each of the three methods will be shown below.

\section{Immediate loading}

The first patient is a 60 -year-old healthy female. A $4 \mathrm{~mm}(\mathrm{w}) \mathrm{x} 3.4 \mathrm{~mm}(\mathrm{p}) \mathrm{x} 13 \mathrm{~mm}(\mathrm{l})$ implant was placed with a $35 \mathrm{Ncm}$ Torque. The definite crown is placed 4.5 months later. 


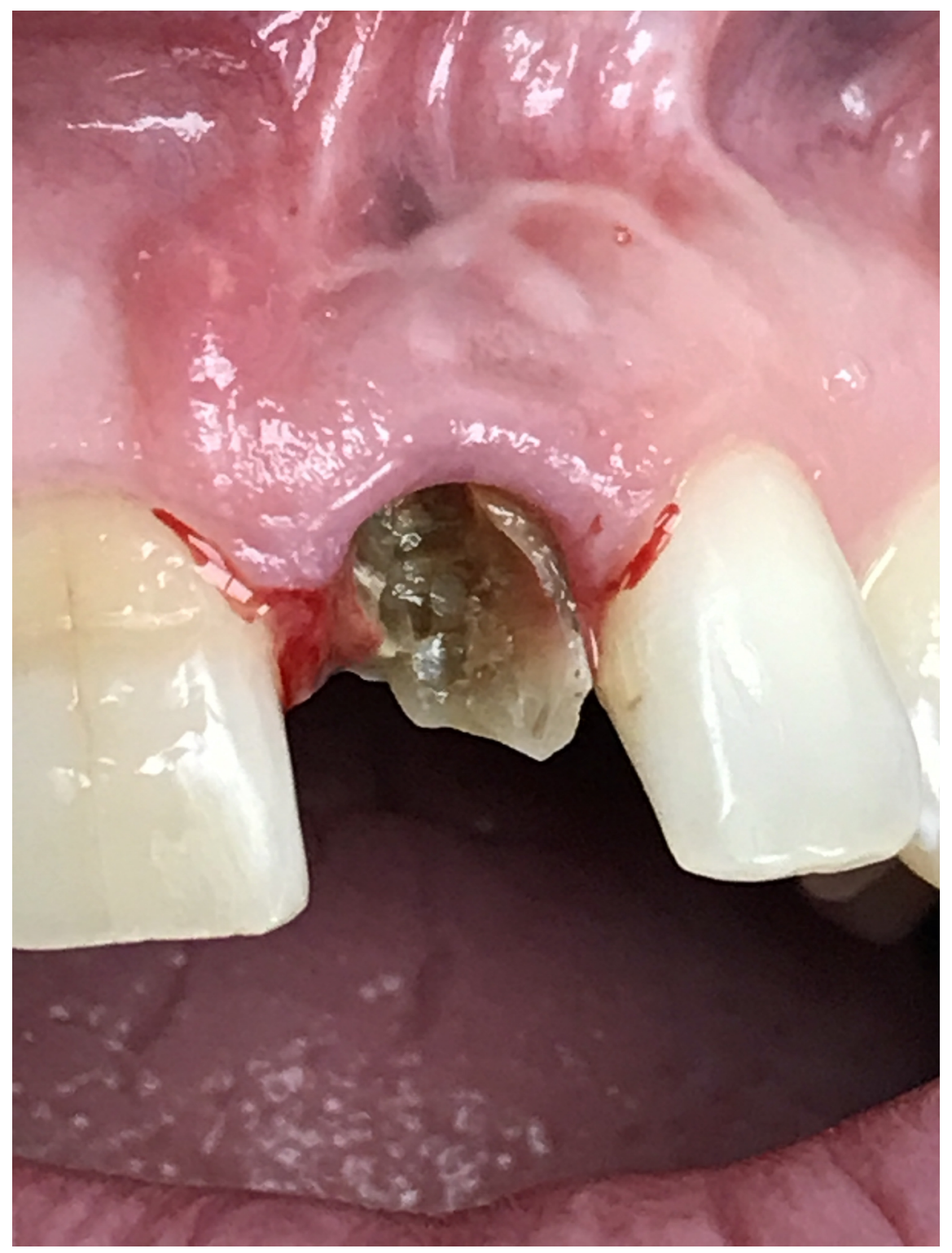




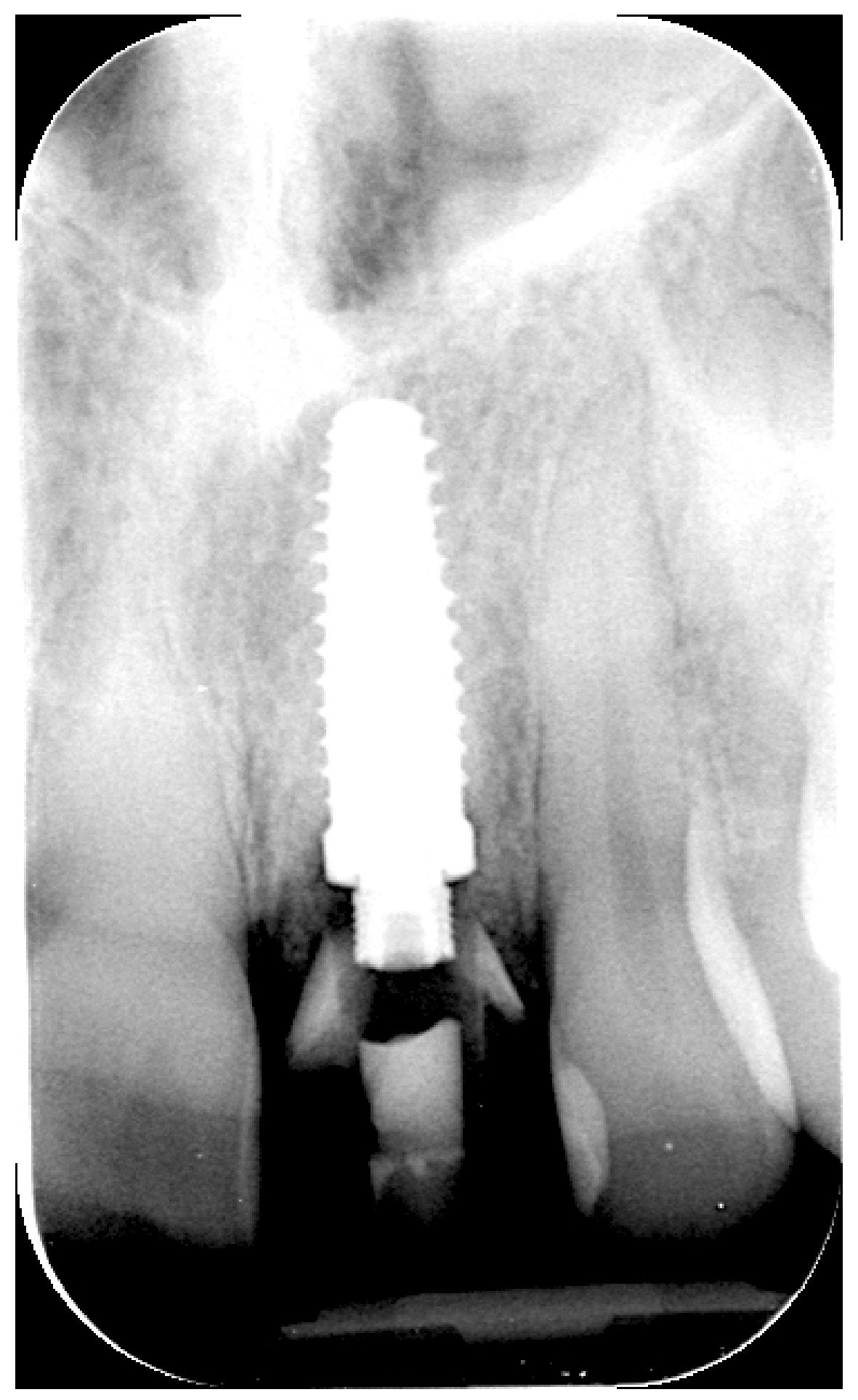

Fig.4, 5. Situation before treatment and implant placed 


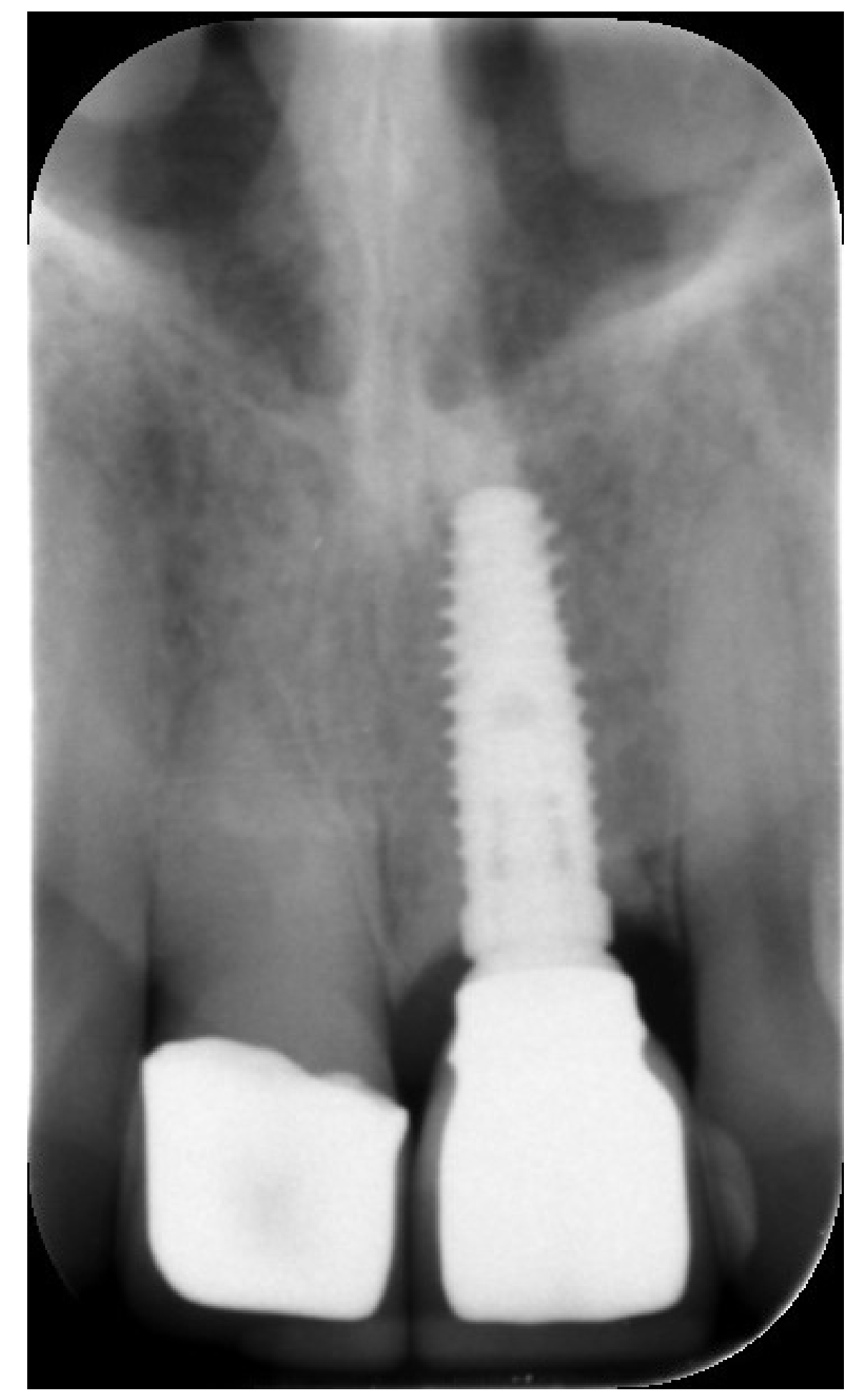




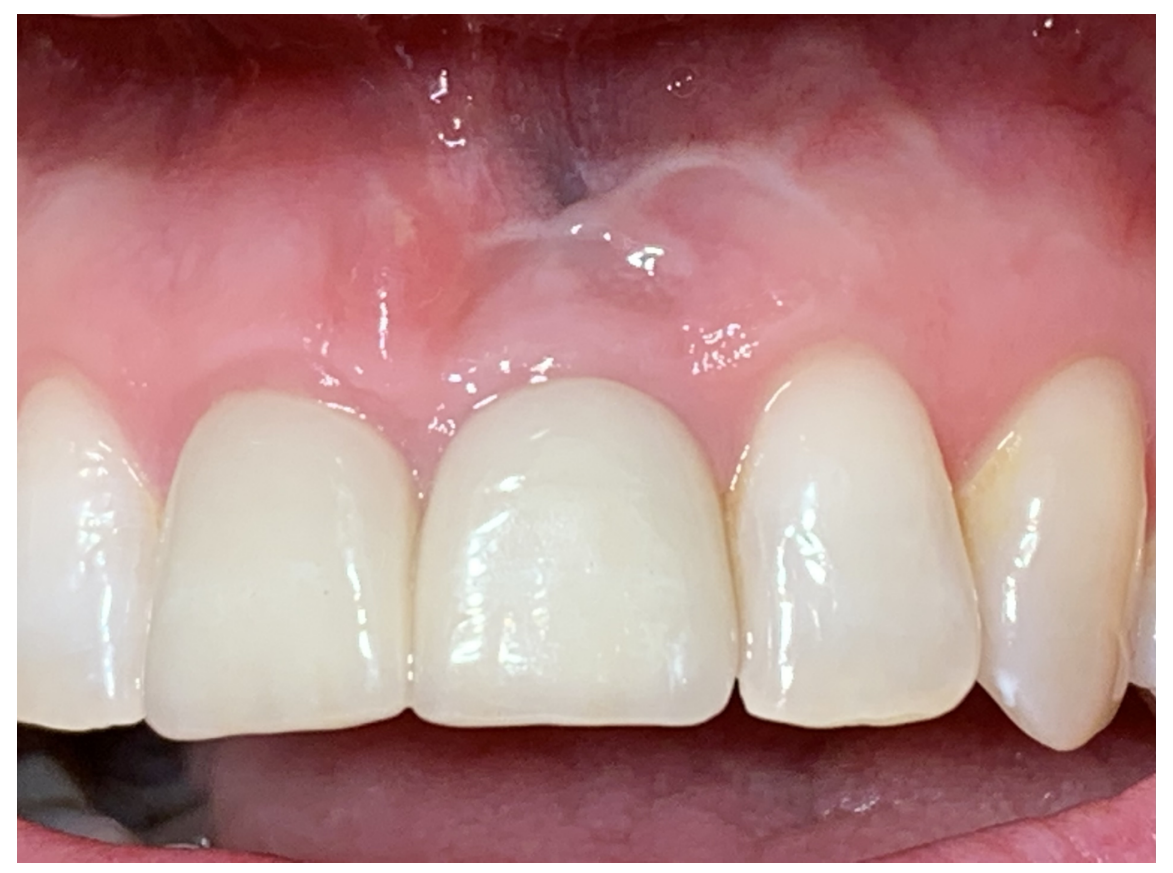

Fig. 6, 7. 3.5 years follow-up

\section{1-phase}

This patient is a 53-year-old male with no medical history. A $4 \mathrm{~mm}(\mathrm{w}) \mathrm{x} 3.4 \mathrm{~mm}(\mathrm{p}) 8.5 \mathrm{~mm}(\mathrm{l})$ implant was placed with a $35 \mathrm{Ncm}$ Torque very close to the maxillary sinu4. The definite crown is placed 5 months after implant placement.

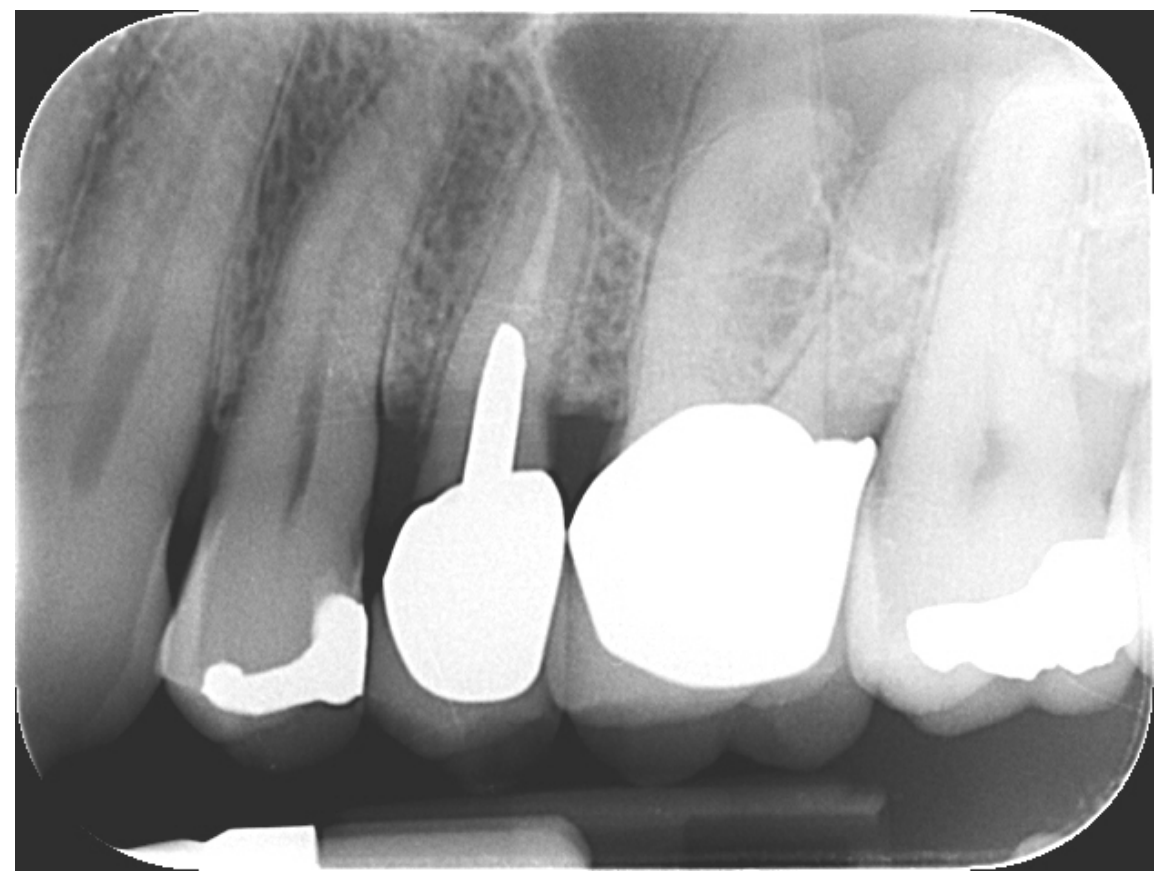




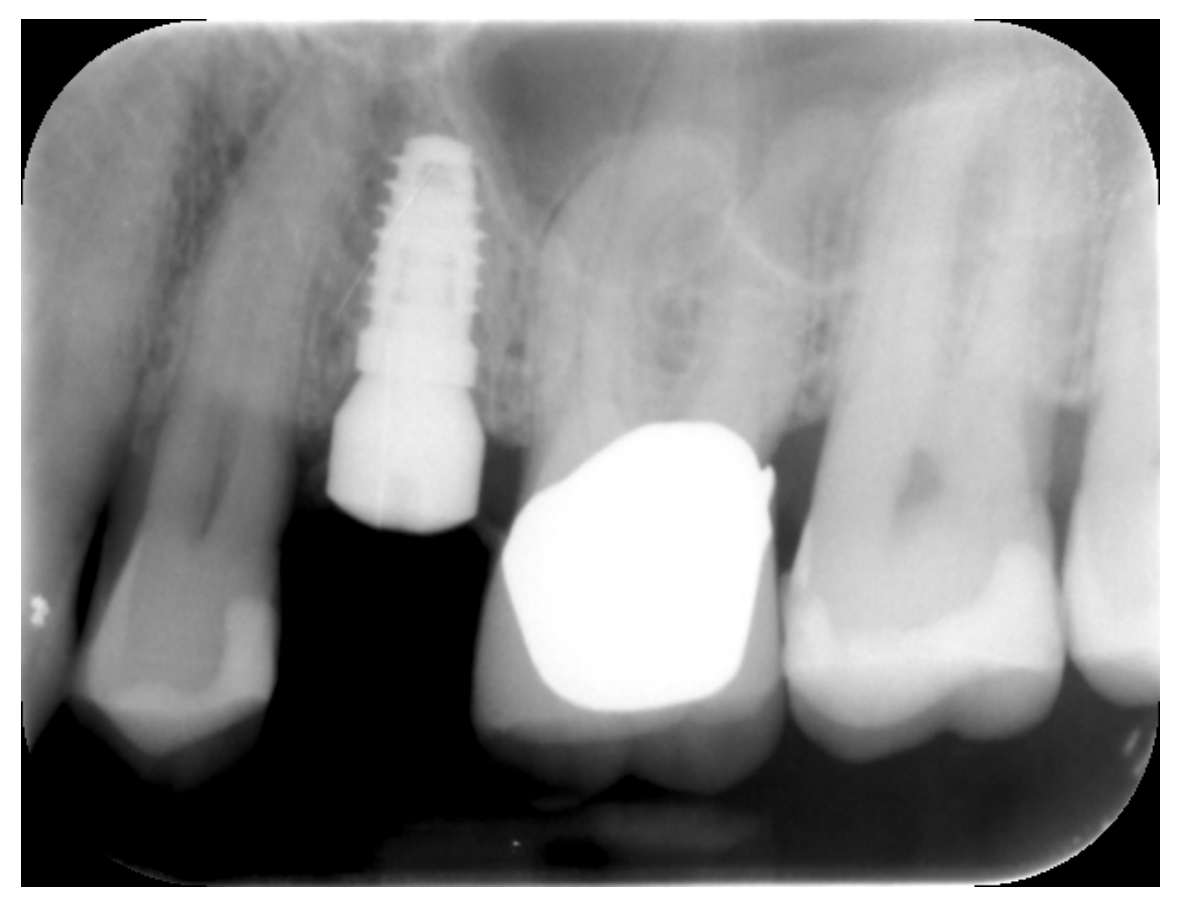

Fig. 8, 9. Situation pretreatment, Implant placement

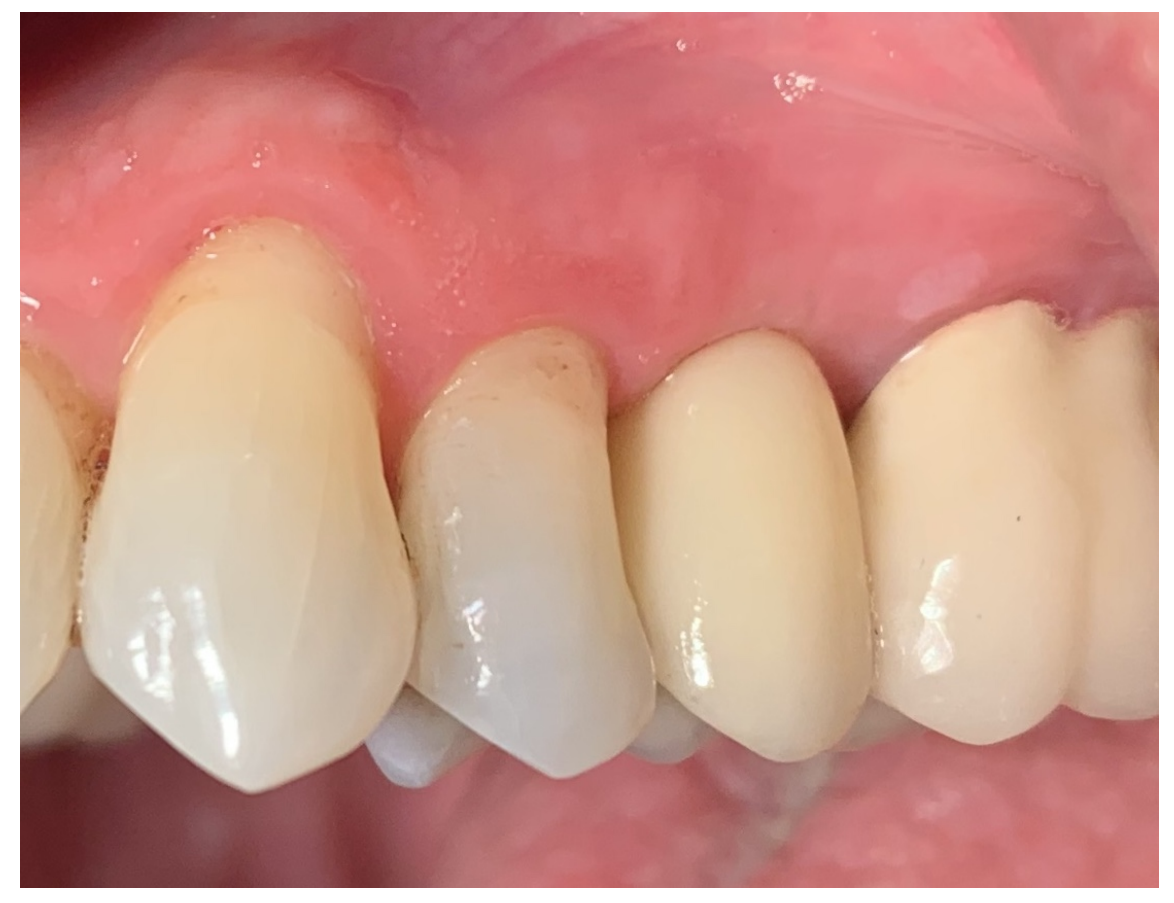




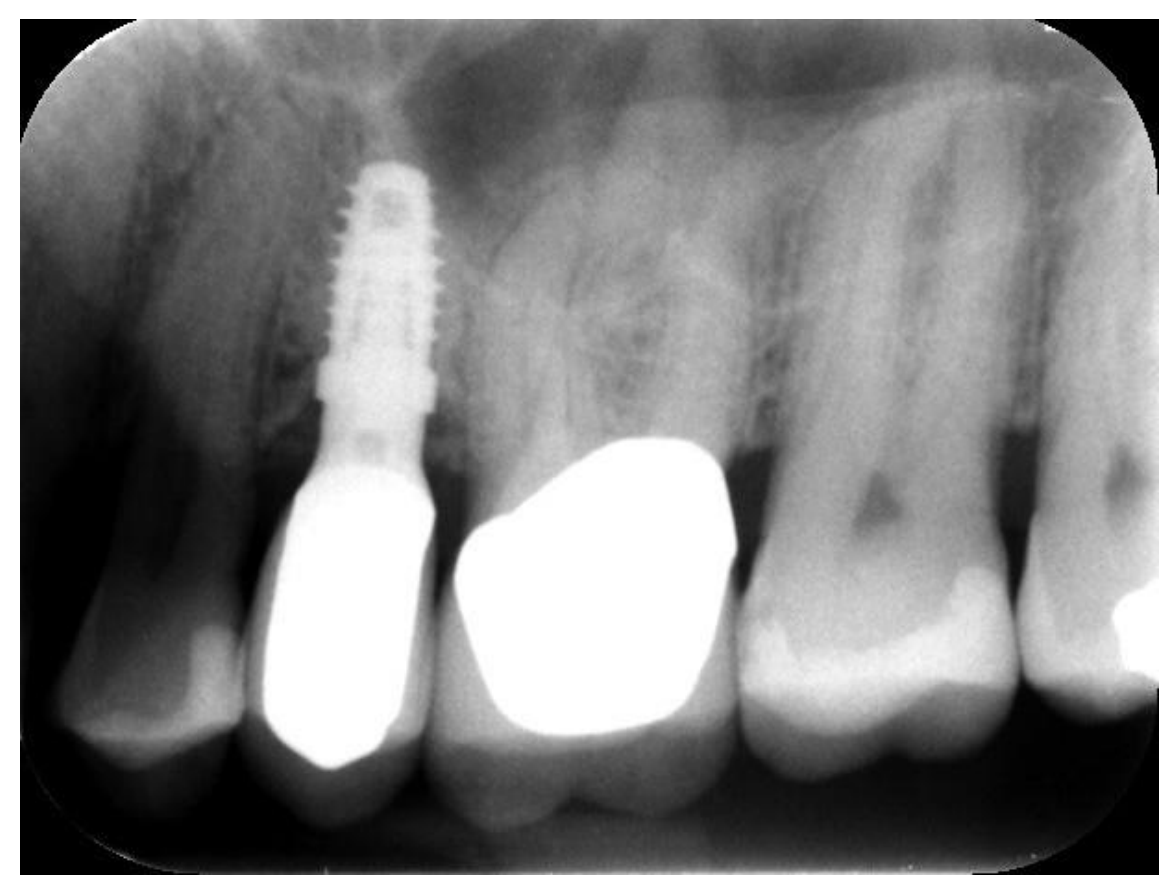

Fig. 10, 11. 24 months follow-up

\section{2-phase}

The 8th patient is $72 \mathrm{yr}$. old male, without medication. After extraction a big bony defect presented itself, which was fully excavated, and a Biomet $3 \mathrm{i}$ implant $5 \mathrm{~mm}(\mathrm{w}) \times 4.1 \mathrm{~mm}(\mathrm{p}) \mathrm{x} 10 \mathrm{~mm}(\mathrm{l})$ was placed at a $25 \mathrm{Ncm}$ Torque. Because of the low initial stability, a 2-phase protocol was followed. The putty was placed to fill the defect. After 3 months the healing abutment was placed. The crown was placed after 9 months, since the implant stability was not sufficient after placing the healing abutment. As a provisional the patient received a partial single tooth prosthesis. 

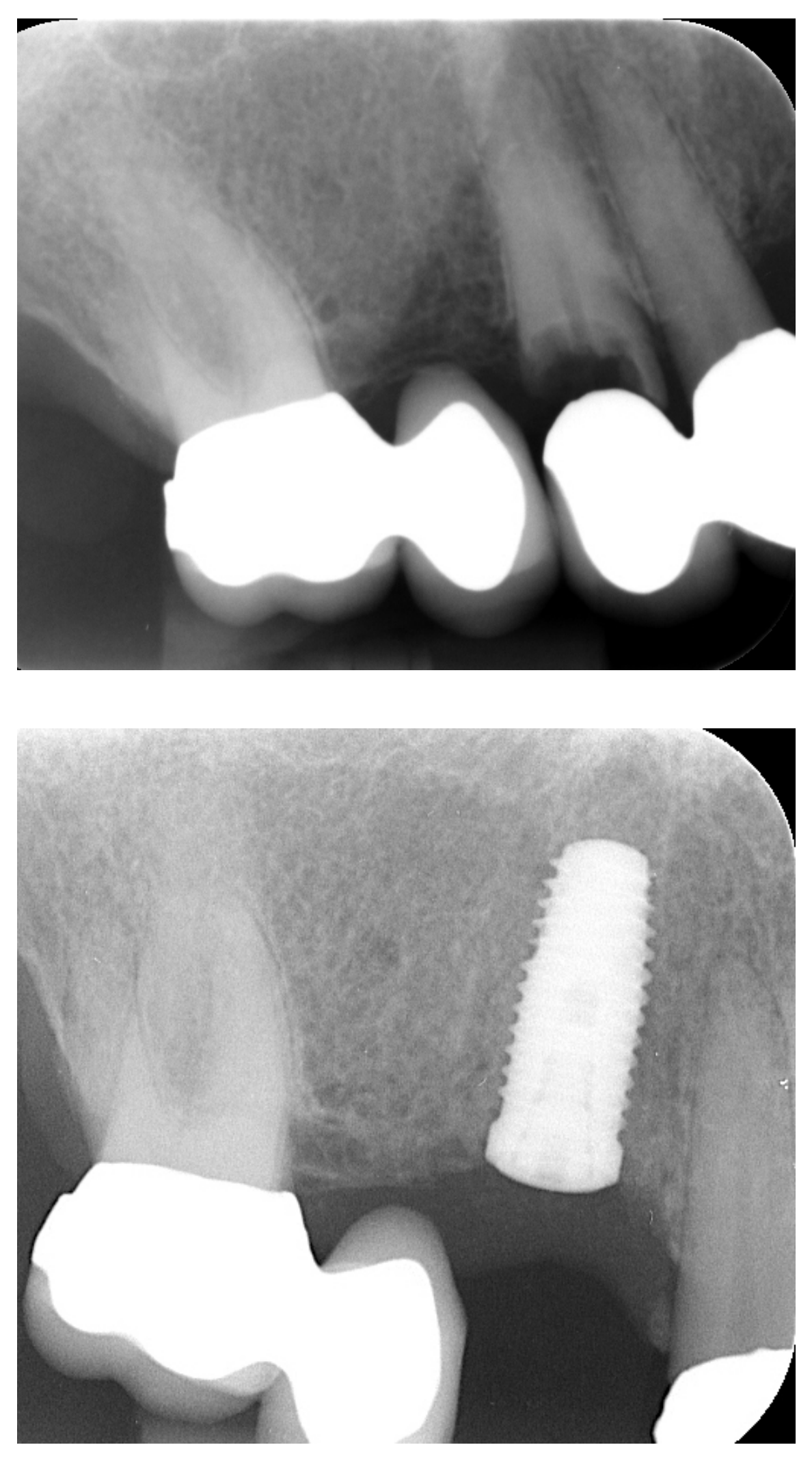
Fig. 12, 13. Clinical situation and implant placement
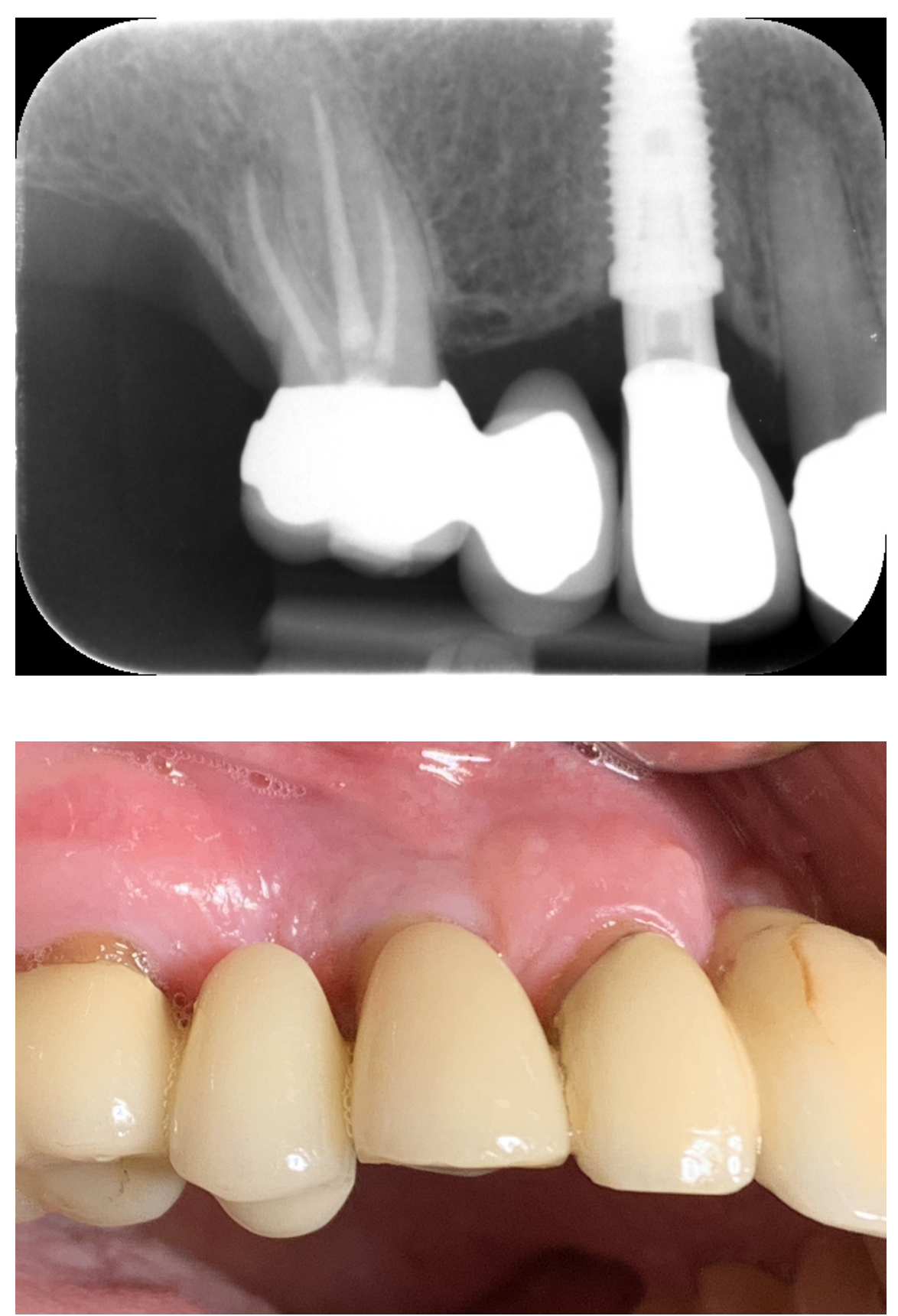

Fig. 14,15 . 16 months Follow-up showing nice pink tissue and bone level

\section{Results}

The results of the 8 patients were evaluated with Pink Esthetic Score (PES). The PES is a tool evaluating the esthetic appearance of the soft tissue around single tooth implants. Each variable is assessed with a 
2-1-0 score, with 2 being the best and 0 being the lowest, which results in a maximum score of 14 . The PES can change over time and therefor is a useful tool for monitoring long-term soft tissue alterations [16].

\begin{tabular}{|c|c|c|c|c|c|c|c|c|c|}
\hline Before treatment & Immediate Ic & & & \multicolumn{2}{|c|}{ 1-phase } & \multicolumn{2}{|c|}{ 2-phase } & \multicolumn{2}{|c|}{ total } \\
\hline case & 1 & 2 & 3 & 4 & 5 & 6 & 7 & 8 & \\
\hline Mesial papilla & 1 & 1 & 1 & 1 & 1 & 1 & 0 & 0 & \\
\hline Distal papilla & 2 & 1 & 1 & 2 & 1 & 1 & 1 & 0 & \\
\hline Level of soft tissue margin & 2 & 2 & 2 & 2 & 2 & 2 & 2 & 2 & \\
\hline Soft tissue contour & 2 & 2 & 2 & 2 & 2 & 2 & 2 & 2 & \\
\hline Alveolar process & 1 & 2 & 2 & 1 & 2 & 2 & 2 & 2 & \\
\hline Soft-tissue color & 2 & 2 & 2 & 2 & 2 & 2 & 2 & 0 & \\
\hline Soft tissue texture & 2 & 2 & 2 & 2 & 2 & 2 & 2 & 0 & \\
\hline PES total & 12 & 12 & 12 & 12 & 12 & 12 & 11 & 6 & 89 \\
\hline & & & & & & & & & \\
\hline After treatment & Immediate lo & & & & & & & & \\
\hline case & 1 & 2 & 3 & 4 & 5 & 6 & 7 & 8 & \\
\hline Mesial papilla & 2 & 1 & 1 & 1 & 1 & 1 & 0 & 0 & \\
\hline Distal papilla & 2 & 1 & 1 & 2 & 1 & 1 & 1 & 0 & \\
\hline Level of soft tissue margin & 2 & 2 & 2 & 2 & 2 & 2 & 2 & 2 & \\
\hline Soft tissue contour & 2 & 2 & 2 & 2 & 2 & 2 & 2 & 2 & \\
\hline Alveolar process & 1 & 2 & 0 & 1 & 2 & 1 & 1 & 1 & \\
\hline Soft-tissue color & 2 & 2 & 2 & 2 & 2 & 2 & 2 & 2 & \\
\hline Soft tissue texture & 2 & 2 & 2 & 2 & 2 & 2 & 2 & 2 & \\
\hline PES total & 13 & 12 & 10 & 12 & 12 & 11 & 10 & 9 & 89 \\
\hline Difference & 1 & 0 & -2 & 0 & 0 & -1 & -1 & 3 & 0 \\
\hline
\end{tabular}

Table 1.

\section{Discussion}

Proper patient selection, atraumatic tooth extraction, palatal implant placement coupled with bone augmentation and careful prosthetic management are all important components in immediate implant placement $[15,17,18]$.

Difference between thick and thin biotypes is not significant, since implants positioned buccally showed three times more recession than ones with a lingual shoulder position[6].

Placing a Bone graft and provisional restauration at the time of anterior tooth extraction increases both the vertical and labial-palatal dimensions between $0.5-1 \mathrm{mmm}$, as compared to not placing a bone graft nor a provisional restoration at the time of flapless immediate post-extraction[19].

All implants placed in extraction sockets without flap elevation showed some negative contour change, however it was minimal compared with studies that elevated full periosteal flaps to extract teeth[18]. The smallest amount of facial-palatal contour change was achieved using bone grafting of the extraction socket at the time of implant placement and stabilization of the graft either by placing a contoured healing abutment or a custom-contoured provisional restoration[18]. A recent study shows minimal soft tissue changes in the horizontal and vertical dimension at single tooth immediate implant placement and provisionalisation in fresh extraction sites in the esthetic zone[8]. The most important feature of this provisional is the transmucosal area which creates an emergence profile for the final esthetic result[17].

The absence of a filler material can contribute to shortening of the marginal gingival margin compromising the aesthetics in immediate implants, especially in the aesthetic region. Therefore, it is suggested that less resorption appears when the space is filled with a low resorption biomaterial, such as bovine bone[4]. Other studies show the same, while grafting extraction sockets does not prevent alveolar bone remodeling, it does minimize buccal bone collapse[17,18].

In these cases, a synthetic putty has been used instead of an xeno- or allograft. Synthetic putty's have shown to preserve good ridge dimension and have favorable bone density values for implant placement as compared to Bio-oss[12].

In these cases, you see a slight decrease in the PES score that can be explained, due to slight sub-crestal implant placement, and case number three had a loose temporary provisional, causing some bone remodeling causing the PES to be lower. Case number 8 had inflamed gingiva due to caries activity, healing perfectly 
after the treatment, causing a higher PES score. In the end there was no difference in PES score before and after treatment (both 11 with 14 being the maximum score), showing nice results overall.

\section{Conclusion}

These case studies are different from the others because of the use of a synthetic putty, and to the best of my knowledge no other article has been written about this. The procedure is simplified because filling the complete gap is easy due to the consistency of the putty, and easy handling with the cartridge.

More research is required to assess the comparison of immediate placement and a synthetic putty in anterior maxillary cases. These cases show a great result in different treatment protocols, and appear to be a good alternative to auto-, xeno- and allograft bone regeneration and immediate placement.

Funding: This case report did not receive external funding

\section{Acknowledgements}

Special thanks to Dr. Peter van der Schoor, Dr. Richard Lazzara and Dr. Barry Levin for their input and support. The author declares that he has no conflict of interest.

(1)- NovaBone Dental Putty, Jacksonville, FL, USA

(2)- Biomet 3i, West Palm Beach, FL, USA

(3)- Protemp 3, 3M ESPE, Seefeld, Germany

(4)- BioHorizons, Birmingham, AL, USA

\section{References}

1. Lazarra RJ, Immediate Implant Placement into extraction sites: Surgical and restorative advantages, Int J Periodontics Rest Dent 198;9:332-343

2. Chu SJ, Saito H, et al. The dual-zone therapeutic concept of managing immediate implant placement and provisional restoration in anterior extraction sockets; Compendium of continuing education in dentistry 2012; vol 33, number 7:524-534

3. Schropp L, Wenzel A, Kostopoulos l, et al. Bone healing and soft tissue contour changes following single-tooth extraction; A clinical and radiographic 12-month prospective study. Int. J Periodontics Restorative Dent 2003;23(4);313-323

4. Denardi RJ, Moreira Melo AC, et al. Bone response after immediate placement of implants in the anterior maxilla: a systematic review. Oral and maxillofacial Surgery 2019; DOI:10.1007/s10006-019-00742-9

5. Januario Al, Duarte WR, Barriviera M. Dimension of the facial bone wall in the anterior maxilla: A cone-beam computed tomography study. J Clin Oral Implant RES 2011;22(10)1168-1171

6. Evans CD, Chen ST, Esthetic outcomes of immediate implant placements, Clin Oral Implants RES 2008;19(1):73-80

7. Araujo MG, Linder E, Lindhe J. Bio-oss collagen in the buccal gap at immediate implants; a 6-month study in a dog, Clin Oral Implants Res 2011;22(1):1-8

8. Amato F, Amato G,Polara G, Spedicato GA, Guided Tissue Preservation: Clinical Application of a New Provisional Restoration Design to Preserve Soft Tissue Contours of Single Tooth Immediate Implant Restorations in the Esthetic Area, The Int. J. of Periodontics \& Restorative Dentistry 2020;6;6:868-879

9. $\quad$ Profeta AC, Prucher GM. Bioactive-glass in periodontal surgery and implant dentistry. Dent Mater J. 2015;34(5);559:71 
10. Gonshor A, Saroff SA, Anderegg CR, Joachim FP, Prasad JACH, Katta S. Histologic and clinical evaluation of a bioactive calcium phosphosilicate bone graft material in postextraction alveolar sockets. International Journal of Oral Implantology and Clinical Research 2011;2,79-84

11. Kotsakis GA, Joachim FP, Saroff SA, Mahesh L, Prasad H, Rohrer MD.Histomorphometric evaluation of a calcium-phosphosilicate putty bone substitute in extraction sockets. Int J Periodontics Restorative Dent $2014 ; 34,233-9$

12. Kotsakis GA, Salama M, Chrepa V, Hinrichs JE, Gaillard P. A randomized, blinded, controlled clinical study of particulate anorganic bovine bone mineral and calcium phosphosilicate putty bone substitutes for socket preservation. Int J Oral Maxillofac Implants 2014;29,141-51

13. Boogaard MJ. Synthetic Putty and Simultaneous Short Implant Placement in Crestal Sinus Lifting Procedures: 13-36 Months Follow-Up: A Case Series. J Dent Rep. 2021;2(1): 1-8.

14. Uppal RS, Pal AS, Gupta G, Kaur R. Evaluating the efficacy of a bioactive synthetic graft material in the treatment of intrabony periodontal defects. International Journal of Contemporary Dentistry 2011;2,5158

15. Goene RJ, Daelen ACL, Mastering aesthetics in post-extraction sites: Journal of Implant and Reconstructive Dentistry; 2013 Vol 4. 1-8

16. Fürhauser R, Florescu D, Benesch T, Haas R, Mailath G, Watzek G. Evaluation of soft tissue around single-toothimplant crowns: the pink esthetic score. Clin. Oral Impl. Res.16, 2005; 639-644

17. Amato f, Polara G, Spedicato GA. Tissue dimensional changes in single-tooth immediate extraction implant placement in the esthetic zone: A retrospective clinical study. Int J Oral Maxillofac. Implants $2018 ; 33 ; 439-447$

18. Tarnow DP, Chu SJ, Salama MA et al. Flapless post extraction socket implant placement in the esthetic zone; Part1. The effect of bone grafting and/or provisional restoration on facial-palatal ridge dimensional change - a Retrospective cohort study. Int J Periodontics Restorative Dent 2014; 34:323-331

19. Chu SJ, Tarnow DP, et al. Flapless post extraction socket Implant Placement, Part 2: The effectss of Bone Grafting and provisional Restoration on Peri-Implant Soft Tissue Height and Thickness- a retrospective Study. Int J Periodontics Restorative Dent 2015;35:803-809 
